\title{
Ambiente Colaborativo Semântico Voltado à Aprendizagem Organizacional para Empresas de Desenvolvimento de Software
}

\author{
André Menolli ${ }^{1}$, Sofia Pinto ${ }^{2}$, Sheila Reinerh ${ }^{3}$, Andreia Malucelli ${ }^{3}$ \\ ${ }^{1}$ Centro de Ciências Tecnológicas - Universidade Estadual do Norte do Paraná (UENP) \\ - Bandeirantes - PR - Brasil \\ ${ }^{2}$ INESC-ID e Instituto Superior Técnico, Universidade de Lisboa - Lisboa - Portugal \\ ${ }^{3}$ Escola Politécnica - Universidade Católica do Paraná - Curitiba, Pr - Brasil \\ menolli@uenp.edu.br, sofia@ontol.inesc-id.pt, sheila.reinehr@pucpr.br, \\ malueppgia.pucpr.br
}

\begin{abstract}
Organizational learning helps companies to improve their processes significantly through the reuse of experiences. However, to make learning organizational possible is not an easy task. Therefore, it is necessary to create means to promote and motivate information sharing and knowledge dissemination. This study aims to propose a semantic collaborative environment based on learning objects and units of learning focused on organizational learning. An initial implementation of the proposed environment was carried out, and an experiment with this environment performed. The results show that the approach is viable to improve organizational learning in software development teams.
\end{abstract}

Resumo. A aprendizagem organizacional auxilia as organizações a melhorar seus processos por meio de reuso de experiências. No entanto, não é uma tarefa trivial alcançar a aprendizagem organizacional. Dessa forma, são necessários meios que facilitem e incentivem o compartilhamento $e$ a disseminação dos conhecimentos. Este trabalho propõe um ambiente colaborativo semântico, baseado em objetos e unidades de aprendizagem, voltado à aprendizagem organizacional. Uma implementação inicial do ambiente proposto foi realizada, e um experimento com este ambiente executado. Os resultados mostram que a abordagem é viável para a melhoria da aprendizagem organizacional em equipes de desenvolvimento de software.

\section{Introdução}

A área de desenvolvimento de software é uma área em que o conhecimento é de vital importância para as corporações, pois os projetos de software são dependentes do conhecimento de seus participantes. Segundo, Rus e Lindvall (2002) os projetos de software são, por natureza, de conhecimento intensivo. Portanto, para a engenharia de software, por ser uma área de processos baseados em conhecimento, é de suma importância aprender com o passado, armazenando e organizando o conhecimento existente de forma a reutilizá-lo, evitando assim erros anteriores. 
Para alcançar o exposto, é necessário mudar práticas organizacionais, reforçar a base de conhecimento das pessoas em relação ao processo de software e compartilhar a base de conhecimento para a organização como um todo. Uma área que pode auxiliar em direção a isto é a aprendizagem organizacional, que é a capacidade ou os processos dentro da organização, destinados a manter ou melhorar o desempenho com base na experiência [Nevis, Di Bella e Gould 1995].

No entanto, alcançar a aprendizagem organizacional não é uma tarefa trivial, pois envolve fatores psicológicos, culturais, administrativos, educacionais, entre outros. Um tema de pesquisa recente que pode apoiar a aprendizagem organizacional, principalmente em empresas de software, são as atuais tecnologias Web, como redes sociais, wikis e blogs, que auxiliam na aprendizagem social. A aprendizagem social centra-se na aprendizagem que ocorre dentro de um contexto social. Considera que as pessoas aprendem umas com as outras, incluindo conceitos como aprendizagem por observação, imitação e modelagem [Ormrod 1999]. Além disso, estas novas tecnologias ou ferramentas colaborativas estão sendo utilizadas por empresas como forma de substituir as intranets empresariais [Capuano, Miranda e Orciuoli, 2009], de forma a fornecer um ambiente em que a comunicação e a colaboração dos trabalhadores aconteça de maneira efetiva, oferecendo um ambiente colaborativo.

No entanto, apenas o uso das ferramentas colaborativas não é suficiente para atingir as características necessárias para que a aprendizagem organizacional ocorra de forma satisfatória, visto que estas ferramentas não têm como objetivo primário a aprendizagem organizacional, mas sim proporcionar ambientes colaborativos, que facilitem a comunicação nas empresas. Assim, além das novas tecnologias e ferramentas baseadas na Web, é necessário organizar as informações de uma forma que possa auxiliar o aprendizado. Uma maneira de fazer isso é usando tecnologias semânticas, como ontologias, de maneira a gerar a aprendizagem organizacional semântica [Sicilia e Lytras 2005]. Outros conceitos que podem ser empregados para melhorar a aprendizagem são conceitos advindos da área educacional, mais especificamente do ensino à distância, tais como, ambientes de e-learning, que são ambientes de ensino não presencial suportados por tecnologia, e objetos de aprendizagem (AO) e unidades de aprendizagem (UA). Dessa maneira, é considerado que a utilização de tecnologias semânticas, e conceitos consolidados da área educacional, podem auxiliar a organizar e contextualizar as informações tanto instrucionalmente como em domínios específicos, o que pode proporcionar um melhor aproveitamento do conhecimento gerado, melhorando assim a aprendizagem dentro das organizações.

Dessa maneira, o objetivo geral deste trabalho que é propor um ambiente colaborativo semântico baseado em objetos e unidades de aprendizagem voltado à aprendizagem organizacional para empresas de desenvolvimento de software. A partir do ambiente proposto, pretende-se responder a seguinte questão de pesquisa:

- "A organização das informações geradas nas empresas, por meio de ferramentas de edição colaborativa, em objetos e unidades de aprendizagem organizacionais, pode auxiliar na aprendizagem dos indivíduos em organizações desenvolvedoras de software?". 


\section{Referencial Teórico}

Esta seção apresenta uma fundamentação teórica dos principais conceitos utilizados como base para propor o ambiente.

\subsection{Aprendizagem Organizacional Semântica}

Em empresas desenvolvedoras de software, existe o desafio de criar e implantar processos que gerem, armazenem, organizem, disseminem e apliquem o conhecimento produzido e utilizado na empresa de modo sistemático, explícito, confiável e acessível à comunidade da organização.

O conceito de aprendizagem organizacional pode auxiliar essa implantação. Segundo Senge et al. (1994) a aprendizagem organizacional pode ser definida como o teste contínuo da experiência e a sua transformação em conhecimento acessível a toda a organização e relevante para seus propósitos fundamentais. No entanto, para uma empresa aplicar os conceitos de aprendizagem organizacional, é necessário utilizar o conhecimento gerado pelos seus membros de forma sistemática e confiável. Para isso, este conhecimento, que muitas vezes é de propriedade exclusiva de apenas um indivíduo, deve ser disseminado. Existem várias ferramentas que podem auxiliar no compartilhamento de informações e na comunicação entre os membros da organização, e, consequentemente, na disseminação do conhecimento. Muitas dessas ferramentas adotam tecnologias semânticas baseadas na Web Semântica [Berners-Lee, Hendler e Lassila 2001]. De acordo com Berners-Lee, a Web Semântica não é separada da Web, mas uma extensão da mesma, o qual um significado bem definido é dado à informação, fazendo com que computadores e pessoas trabalhem em cooperação.

A Web semântica é uma área que tem tido um grande crescimento e tem sido alvo de inúmeras pesquisas. As técnicas, conceitos e aplicações da Web semântica vêm sendo aplicadas em diversas áreas, fazendo com que novas linhas de pesquisa sejam criadas. Como exemplo surgiu os sistemas educacionais baseados em Web semântica [Capuano, Miranda e Orciuoli 2009] e a aprendizagem organizacional semântica. Aprendizagem Organizacional Semântica é um conceito que estende a noção de aprendizagem organizacional na dimensão tecnológica [Sicilia e Lytras 2005], ou seja, não é apenas a aplicação de recursos tecnológicos semânticos para atingir a aprendizagem organizacional. É o uso de tecnologias que fornecem uma representação do conhecimento compartilhado sobre um domínio e contexto da organização, com o intuito de mediar e intensificar as atividades de aprendizagem.

Portanto, a aprendizagem organizacional semântica pode ser considerada a aplicação de recursos da Web semântica para promover a aprendizagem organizacional dentro de uma empresa [Menolli, Malucelli e Reinehr 2011]. Segundo Sicilia e Lytras (2005), as tecnologias que podem promover a Web semântica, devem ser aplicadas não só para melhorar os processos de aprendizagem, mas como uma ferramenta de propósito para conduzir mudanças no comportamento.

\subsection{Ferramentas Aplicadas à Organizações}

Para que a aprendizagem organizacional ocorra é necessária uma mudança de cultura, melhoria da comunicação e principalmente a adaptação da empresa e membros às novas 
realidades. Cada empresa deve avaliar as tecnologias disponíveis e verificar qual é a mais adequada para conduzir ou facilitar a aprendizagem na organização. Além das tecnologias tradicionais já utilizadas por empresas, como intranets, ferramentas de comunicação online, repositórios de dados compartilhados e outras tecnologias que dão suporte a comunicação e armazenamento do conhecimento, existem novas tecnologias que podem ser aplicadas na busca da aprendizagem organizacional. Dentre essas tecnologias, as tecnologias Web sociais recentes se destacam, pois, segundo Rech e Ras (2008), facilitam a colaboração distribuída, estimulando a livre reutilização da informação, de experiências, ou produtos, e apoia os trabalhadores do conhecimento a lidar com a sobrecarga de informação, integrando e reutilizando informações espalhadas por diversas fontes de conteúdo. Dentre as recentes tecnologias da Web, as principais utilizáveis para a aprendizagem organizacional são [Rech e Ras 2008]: (1) Wikis: permitem a colaboração de pessoas distribuídas visando compartilhar ideias, experiências e links; (2) Web Blogs: são plataformas de distribuição de conteúdo para compartilhar notícias sobre um tema específico e são usados para o compartilhamento de conhecimento, experiências ou documentos; (3) Sistemas de Discurso: são os sucessores dos quadros de avisos e são usados sistematicamente para discutir temas relevantes, com ou sem a ajuda dos moderadores, e obter experiências valiosas em um ambiente distribuído, mas participativo; (4) Folksonomias: são indexações criadas com base em tags livremente designadas pelos usuários de um sistema.

Além das tecnologias Web sociais, outras tecnologias podem ajudar na construção de uma plataforma semântica para a aprendizagem organizacional, como ontologias, que abstrai o conhecimento de um determinado domínio e representa-o formalmente por meio da conceitualização, expressa em lógicas formal [Gruber 1995].

\subsection{Conceitos Educacionais}

O ambiente proposto baseia-se em alguns conceitos educacionais, como objetos e unidades de aprendizagem. Cada um desses conceitos educacionais é descrito a seguir, no entanto foram adaptados para a realidade das organizações, uma vez que a aprendizagem no ambiento educacional e organizacional apresenta diferenças significativas [Menolli, Malucelli e Reinehr 2011].

\subsubsection{Padrões de Objetos de Aprendizagem}

Objetos de aprendizagem (OAs) são definidos como qualquer recurso reprodutível e endereçável digital ou não digital usado para executar atividades de aprendizagem ou atividades de apoio [IMS 2003]. Esses objetos são definidos por metadados, que descrevem o recurso educacional, facilitando assim a interoperabilidade e reuso entre a diferentes plataformas educacionais.

Existem várias especificações de metadados na literatura. Normalmente, os metadados são definidos por associações, tais como IEEE (1484/12/01 Standard for Learning Object Metadata) e ISO (SC 36 WG 2 - Information Technology for Learning, Education, and Training), que criaram grupos de trabalhos para desenvolver propostas de estruturação e categorização de objetos (metadados), com o objetivo de apoiar a catalogação de OAs, de forma a serem devidamente recuperados e reutilizados. Assim, estes metadados representam uma maneira de organizar os dados dos OAs, 
permitindo a comunicação entre os diferentes ambientes de computação, bem como a sua acessibilidade e usabilidade, além de garantir a sua interoperabilidade. Alguns exemplos de padrões são o Learning Object Metadata (LOM) [IEEE 2002] e Sharable Content Object Reference Model (SCORM), que é um modelo de referência que foca a reusabilidade e a interoperabilidade dos objetos de aprendizagem [ADL 2004].

\subsubsection{Unidades de Aprendizagem}

O outro conceito educacional utilizado neste trabalho é a unidade de aprendizagem. Uma unidade de aprendizagem é um conceito mais abrangente do que um objeto de aprendizagem, pois define um módulo geral para um processo de aprendizagem, como um curso, por exemplo [IMS 2003]. A unidade de aprendizagem é composta basicamente por duas partes, o learning design e os recursos físicos [IMS, 2003].

O learning design define a estrutura de uma unidade de aprendizagem, e é criado utilizando alguma Linguagem de Modelagem Educacional (LME), que são modelos de informação semântica e agregações, que descrevem, de um ponto de vista pedagógico, o conteúdo assim como as atividades educacionais [Amorim et al. 2006]. Uma das principais linguagens de modelagem educacional é o IMS Learning Design (IMS LD) [IMS 2003], que dá suporte ao uso de diferentes abordagens de ensino/aprendizagem, tais como: comportamentalistas, cognitivistas e construtivistas.

A especificação IMS LD permite representar qualquer unidade de aprendizagem e pode ser baseada em uma ampla gama de técnicas pedagógicas. O IMS LD permite que uma unidade de aprendizagem seja modelada como content packages. Os content packages são a estrutura física dos cursos. A IMS Content Packaging Specification descreve como os recursos digitais podem ser organizados em unidades lógicas de aprendizagem chamados de content packages [IMS 2004].

\section{Trabalhos Relacionados}

No estudo apresentado Menolli, Reinehr e Malucelli (2013a) é exposta uma revisão sistemática que mostra como os conceitos de aprendizagem organizacional estão sendo aplicados nos trabalhos de engenharia de software nos últimos anos. Percebe-se que muitos estudos se concentram nos processos de software, além disto, os conceitos de lições aprendidas e fábrica de experiências normalmente são bastante utilizados nos trabalhos de aprendizagem organizacional na área de engenharia de software, e além disto, há muitas pesquisas com foco em desenvolvimento distribuído.

Percebe-se também, que o uso de ferramentas e tecnologias semânticas, a fim de aprimorar o aprendizado em projetos de engenharia de software vem ocorrendo. Por exemplo, Biasutti e EL-Deghaidy (2012) mostram uma pesquisa em que uma wiki foi utilizada como ferramenta didática no desenvolvimento de processos de gestão do conhecimento. Estudantes utilizaram a colaboração online para planejar projetos interdisciplinares, considerando os princípios construtivistas sociais da aprendizagem. Outro estudo com foco na wiki é apresentado por Tseng e Huang (2011). Neste estudo, os conteúdos, valores técnicos e sociais da Wikipedia são analisados para explorar a sua influência sobre a partilha de conhecimento e desempenho no trabalho. 
No entanto, verifica-se que conceitos advindos da área educacional não são frequentemente utilizados para prover aprendizagem organizacional na área de engenharia de software. As pesquisas que utilizam estes conceitos normalmente são focadas no campo educacional. Por exemplo, o trabalho de Zouaq e Nkambou (2009), apresentam uma abordagem que permite a capitalização dos recursos pedagógicos existentes, criando primeiro metadados de conteúdo baseado na mineração de texto e processamento de linguagem natural, para ser possível criar dinamicamente objetos de aprendizagem.

\section{Ambiente Proposto}

Para que ocorra aprendizagem de forma efetiva dentro das empresas são muitos os requisitos necessários, como é apresentado por Menolli, Malucelli e Reinehr (2011). Para que todas as características apresentadas sejam atingidas, o aprendizado deve ser guiado de forma que se utilizem os preceitos das mais diversas teorias de aprendizagem.

Uma das principais características do ambiente proposto é o compartilhamento do conhecimento, uma característica já há muito tempo notoriamente conhecida como um dos principais fatores da aprendizagem organizacional, e alcançada por meio da utilização de ferramentas de colaboração. Propõe-se também que todo os conteúdos gerados por ferramentas colaborativas sejam organizados em ontologias. A ontologia usada no ambiente é apresentada na seção 4.1 e arquitetura do ambiente, com uma descrição detalhada de cada componente, é apresentada na seção 4.2.

\subsection{Unit of Organizational Learning Ontology (UOLO)}

Uma ontologia, denominada, Unit of Organizational Learning Ontology (UOLO) [Menolli et al. 2014], foi definida para orientar a organização de materiais produzidos em diferentes ferramentas colaborativas. A UOLO, apresentada na Figura 1, visa ajudar a organizar conteúdos criados nas empresas em objetos e unidades de aprendizagem, e foi desenvolvida em três módulos principais: (1) objetos de aprendizagem organizacionais [Menolli, Reinehr e Malucelli 2012a]; (2) learning design; e (3) content package.

A UOLO originalmente começou por considerar os principais conceitos da Ontology for Organizational Learning Object [Menolli, Reinehr e Malucelli 2012a]. Este módulo é baseado no padrão LOM [IEEE 2002] e fragmentos da ontologia FOAF (Friend of a Friend) [FOAF 2010]. Este módulo representa objetos de aprendizagem organizacionais, tendo em conta as propriedades educacionais e organizacionais. A Figura 1 (Parte A) mostra os conceitos deste módulo.

O learning design, tem o objetivo de auxiliar na organização de materiais anteriormente produzidos, de forma a melhorar a sua compreensão. Assim, por meio de um learning design, os materiais podem ser organizados como um curso, por exemplo. Tomando o IMS LD [IMS 2003] como um ponto de partida, o módulo de learning design foi definido como mostrado na Figura 1 (parte B).

A última parte do UOLO é a definição dos content packages, que descreve a estrutura física do curso definido por um learning design. Para definir os content 
packages, foi utilizado como base o IMS Content Packaging Specification [IMS 2004], e esta parte da ontologia é mostrada na Figura 1 (Parte C).

A Figura 1 representa a estrutura completa de UOLO. Uma unidade de aprendizagem está relacionada tanto a um learning design, que descreve a estrutura do curso, quanto a um content package, que descreve o conjunto de recursos, que é composta de um ou vários objetos OLOs.

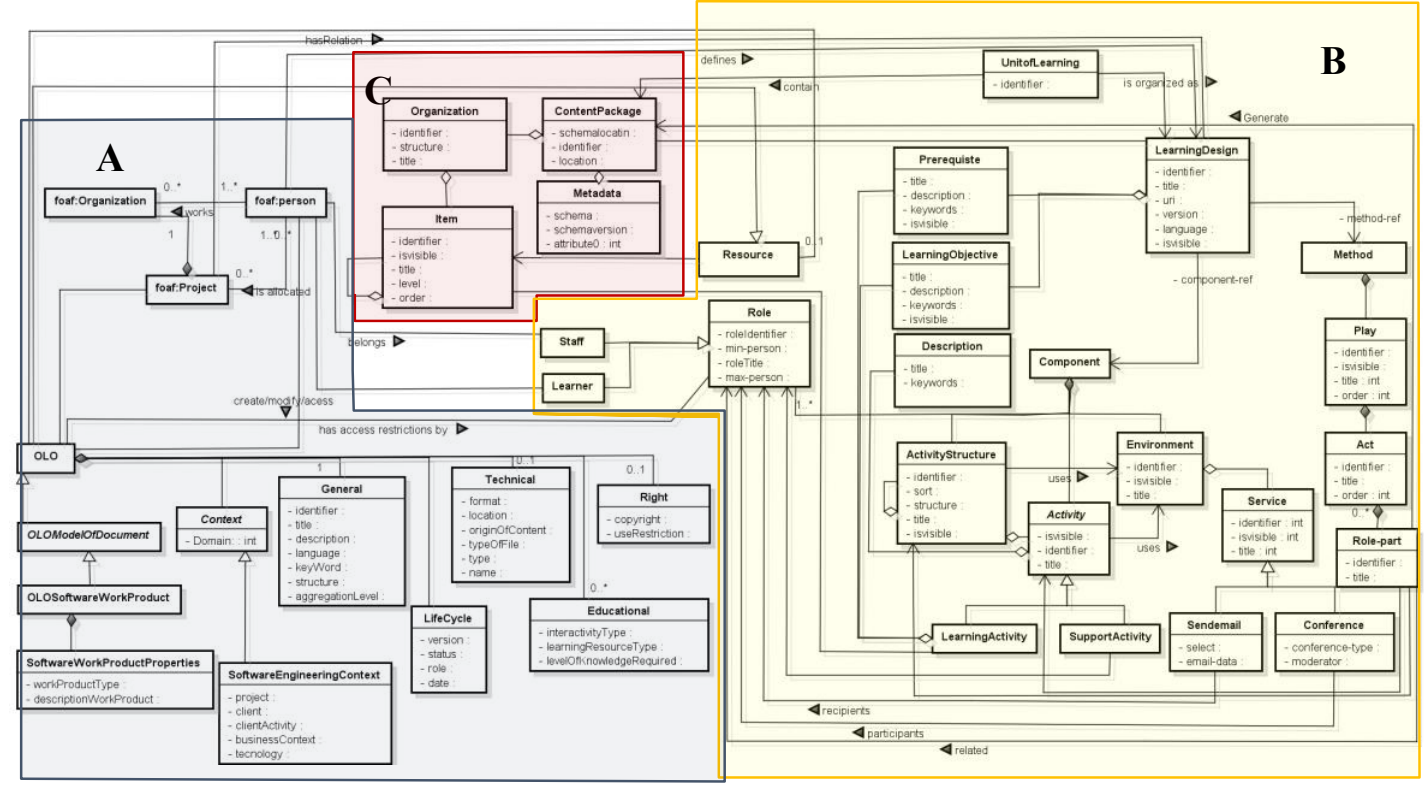

Figura 1. Conceitos, Taxonomia e Relações da UOLO

\subsection{Arquitetura do Ambiente}

Esta seção apresenta uma arquitetura que reúne e organiza os componentes do ambiente proposto, para criar um ambiente de colaboração social semântico. A Figura 2 apresenta uma visão geral da arquitetura proposta para o ambientem, e a arquitetura é subdividida em duas camadas principais: a camada de aplicação e a camada de memória organizacional.

\subsubsection{Camada de Aplicação}

A Camada de Aplicação é responsável pela interação com o usuário e fornece subsídios para a inserção de conteúdos, criação de unidades de aprendizagem, além de apresentar uma interface para realizar buscas na memória organizacional. Esta camada é composta de três componentes com funções distintas:

(i) Componentes da Interface com o Usuário: estão as ferramentas colaborativas que os usuários finais interagem, como wiki, blogs entre outras. Estas ferramentas são configuradas de acordo com um metamodelo chamado Meta model of Instructional Context (MIC), que tem como objetivo organizar as ferramentas para promover a integração da informação, respeitando o contexto do domínio do conhecimento, bem como o papel instrucional dos conteúdos;

(ii) Interface de Unidade de Aprendizagem: por meio deste componente um especialista pode criar de forma semi-automática as unidades de aprendizagem 
organizacionais, utilizando os materiais inseridos pelos usuários na interface com o usuário;

(iii)Busca de Objetos e Unidades de Aprendizagem: permite que pesquisas por objetos de aprendizagem organizacionais e unidades de aprendizagem organizacionais sejam realizadas na memória organizacional.

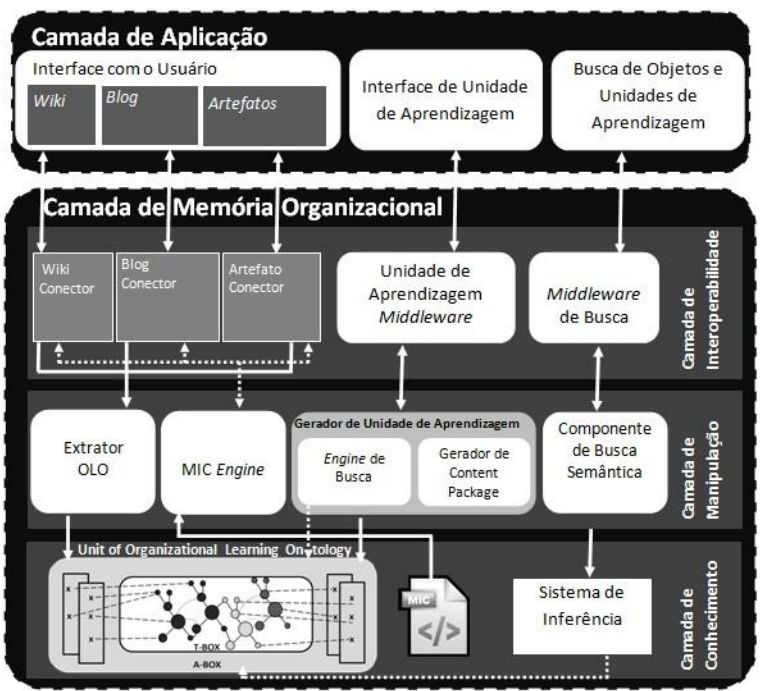

Figura 2. Arquitetura Proposta. Adaptado de [Menolli, Malucelli e Reinehr 2011]

\subsubsection{Camada de Memória Organizacional}

A Camada de Memória Organizacional é responsável pelo armazenamento de todo o conhecimento gerado na camada de aplicação, assim como a manipulação desse conteúdo de forma que possa ser organizado de acordo com a representação definida UOLO. Suas respectivas subcamadas são: interoperabilidade, manipulação e conhecimento.

A Camada de Interoperabilidade fornece um mecanismo de extensibilidade para permitir a incorporação de novas ferramentas na arquitetura descrita e organiza as ferramentas presentes na interface com o usuário, de maneira a respeitar as definições especificadas no MIC.

A Camada de Manipulação é responsável por manipular os dados das camadas superiores e encaminhá-los à camada de conhecimento. Esta camada apresenta quatro componentes com funções distintas.

(i)MIC Engine: é responsável por fornecer informações sobre a estrutura da MIC aos conectores das ferramentas presentes na interface com o usuário.

(ii)Extrator OLO: cria instâncias de objetos de aprendizagem organizacionais na UOLO a partir dos conteúdos inseridos nos componentes de interface com o usuário.

(iii)Gerador de Unidade de Aprendizagem: esse componente é subdividido em dois subcomponentes. Engine de Busca: lê um esquema de learning design, e para cada learninActivity presente no learning 
design, retorna uma lista de objetos de aprendizagem organizacionais correspondentes. Gerador de Content Pacakage: é responsável por popular a UOLO, criando instâncias dos content package e learning design na ontologia.

(iv)Componente de Busca Semântica: organiza as consultas para o motor de inferência e controla o conhecimento inferido organizando os resultados, tratando erros, exceções e comportamentos inesperados durante a execução.

A última camada da arquitetura proposta é a Camada de Conhecimento. Esta camada fornece um sistema de inferência para gerenciar o conhecimento compartilhado entre os aplicativos e o comportamento do sistema, recebendo as informações fornecidas pelas diferentes ferramentas e as organiza em objetos de aprendizagem organizacionais e posteriormente em unidades de aprendizagem organizacionais utilizando a UOLO.

\subsection{Implementação do Ambiente}

Usando a arquitetura proposta, a primeira implementação do ambiente foi realizada. Nesta primeira implementação, apenas ferramenta wiki foi utilizada na camada de aplicação. O ambiente implementado permite que a partir de conteúdo inserido por usuários na ferramenta social, sejam criadas semi-automaticamente unidades UOLO, como mostrado no esquema da Figura 3. Esse esquema é dividido em três partes principais, Pré-processamento, Gerador de Curso e Componente de Busca.

\subsubsection{Pré-Processamento}

O pré-processamento é a primeira etapa para gerar as unidades de aprendizagem organizacionais. Nesta etapa, o usuário insere os conteúdos na wiki, e a partir destes conteúdos os OLOs são gerados. Uma wiki privada, chamada SSEW (Semantic Software Engineering Wiki) foi utilizada como ferramenta social.

Após o conteúdo ter sido inserido na ferramenta wiki, o extrator OLO extrai informações sobre cada página e as define como um OLO, usando a abordagem apresentada por [Menolli, Reinehr e Malucelli 2012b]. Como mostrado na Figura 3, a UOLO está dividida em duas partes: a T-Box que contém um conjunto de axiomas terminológicos [Baader, Horrocks e Sattler 2008], e a $A$-Box que contém um conjunto de asserções descritas pela $T$-Box. Deste modo, o extrator OLO preenche a $A-B o x$ da UOLO com informações sobre os objetos de aprendizagem organizacionais (Figura 1 Parte A), criando indivíduos OLO na $A$-Box.

\subsubsection{Gerador de Curso}

O gerador de cursos é a etapa responsável por gerar os content packages que compõem as unidades de aprendizagem organizacionais. Primeiramente, um especialista é responsável por criar um learning schema, que define a estrutura da unidade de aprendizagem como mostrado na Figura 3 (1). Em seguida, a Engine de Busca lê a estrutura do curso definida no learning schema e procura na UOLO por OLOs que sejam candidatos para cada uma das atividades de aprendizagem definidas para o curso. Assim, a Engine de Busca retorna uma lista de OLOs candidatos para cada learning activity. Depois disso, o especialista escolhe nesta lista, o melhor objeto de 
aprendizagem organizacional para cada atividade de aprendizagem, Figura 3 (2). Uma vez selecionados os OLOs para cada learning activity definida no learning schema, o Gerador de Content Package acessa o learning schema para criar os content packages. Este componente também popula a A-Box da UOLO com o learning schema, apresentado na Figura 1 (Parte B) e com os content packages criados, apresentados na Figura 1 (Parte C).

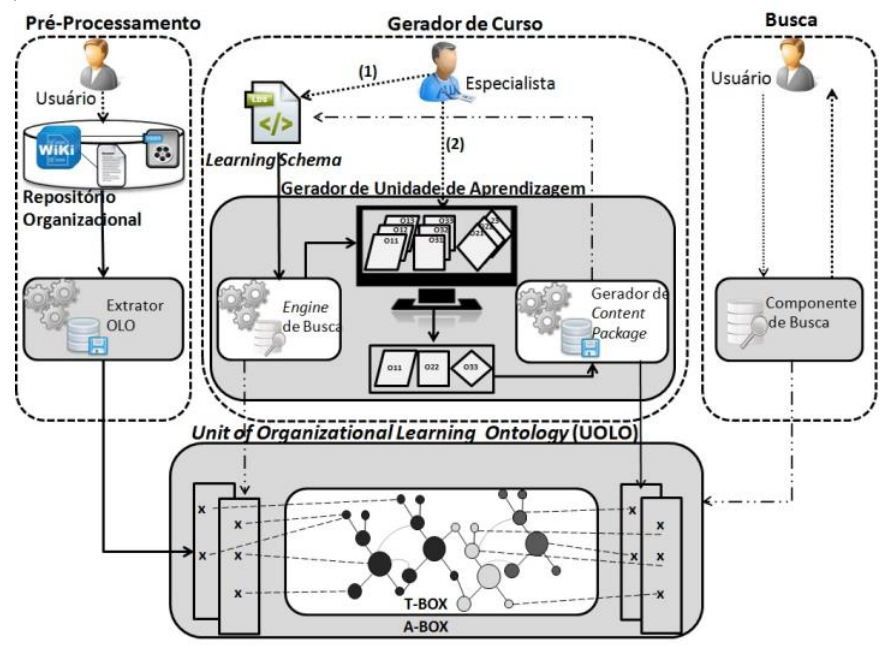

Figura 3. Esquema para Implementar o Ambiente

\subsubsection{Componente de Busca}

Após a ontologia ser preenchida, os usuários podem realizar pesquisas por objetos de aprendizagem organizacionais ou unidades de aprendizagem organizacionais.

\section{Experimento}

O ambiente foi implementado utilizando como ferramenta social uma wiki, e a partir dos conteúdos inseridos nessa wiki, objetos de aprendizagem organizacionais e unidades de aprendizagem organizacionais foram concebidos. Assim um experimento foi realizado, de forma a analisar o impacto que o uso do ambiente tem sobre os membros de uma equipe de desenvolvimento de software em comparação com o simples uso da wiki, que contém o mesmo conteúdo. Este experimento tem como principal objetivo analisar sob quais aspectos o uso do ambiente pode auxiliar os membros de uma equipe de desenvolvimento de software.

Os critérios para avaliar o experimento, foram definidos com base em um experimento anterior já realizado [Menolli, Reinerh e Malucelli, 2013b], e foram definidos três critérios a serem analisados: 1) Tempo de desenvolvimento, 2) Qualidade da solução e 3) Aquisição do conhecimento.

Para realização do experimento, foram selecionados dez participantes, alunos de graduação que foram divididos em dois grupos. Os participantes foram solicitados a realizar três manutenções, todas aplicações de padrões de projeto em um software existente. Na realização do experimento, os participantes podiam acessar conteúdos para auxiliá-los a resolver as tarefas propostas, de acordo com o grupo que pertenciam: 
- Grupos 1 - participantes usando o ambiente (os usuários puderam usar a SSEW e a pesquisa por objetos e unidades de aprendizagem organizacionais) para ajudálos a implementar a tarefa desejada.

- Grupo 2 (Grupo de Controle) - participantes apenas usando a SSEW para ajudálos a implementar a tarefa desejada.

Ao final de cada manutenção os participantes responderam questionários sobre a implementação proposta. Além disso, um especialista da área acompanhou todo o experimento e também preencheu um questionário para avaliar o trabalho realizado pelos participantes de acordo com critérios específicos.

\subsection{Resultados}

O tempo de desenvolvimento para as três manutenções, em média sempre foi menor para quem teve acesso às unidades de aprendizagem. O tempo médio para realizá-la foi de 94 minutos para quem teve acesso as unidades, enquanto que para quem não teve acesso, o tempo médio foi de 116,6 minutos, uma diferença de $24 \%$. Quem não teve acesso às unidades, o tempo total de desenvolvimento das três manutenções foi em média $15 \%$ superior em relação aos participantes que tiveram acesso. Portanto, pode-se concluir que os desenvolvedores que têm acesso aos conteúdos, organizados como objetos e unidades de aprendizagem organizacionais, solucionam as tarefas mais rapidamente do que os que não têm acesso.

A qualidade da solução foi definida usando critérios objetivos em cada uma das manutenções. Para cada manutenção, o especialista decidiu se o participante havia implementado corretamente, a partir de critérios claros e objetivos, como por exemplo, se foi retirado determinado código de determinada classe; se foi criada uma nova classe e assim por diante.

Observando a Figura 4, percebe-se que os participantes que tiveram acesso às unidades e objetos de aprendizagem, nas três manutenções conseguiram implementar as melhorias propostas, e sempre conforme o esperado ou com pequenas modificações. Para os participantes que não tiveram acesso aos objetos e unidades, $40 \%$ das manutenções foram implementadas de forma fora do esperado ou parcialmente implementada. Por outro lado, $60 \%$ das manutenções executadas pelos participantes que tiveram acesso às unidades e objetos de aprendizagem organizacionais foram implementadas conforme o esperado. Pode-se concluir que a utilização dos conteúdos, organizados como objetos e unidades de aprendizagem organizacionais, auxilia a melhorar a qualidade das soluções nas tarefas propostas.

Para verificar a aquisição do conhecimento, vários aspectos foram analisados. Foi levado em conta a qualidade da solução o nível de entendimento que o participante teve sobre a manutenção proposta e a sua opinião sobre a complexidade da manutenção.

Analisando a Figura 5, percebe-se que os participantes que tiveram acesso às unidades e objetos de aprendizagem organizacionais, tiveram um entendimento melhor dos padrões propostos nas três manutenções. Além disso, os participantes que não tiveram acesso as unidades e objetos, no geral, consideram as manutenções mais complexas do quem teve acesso. 


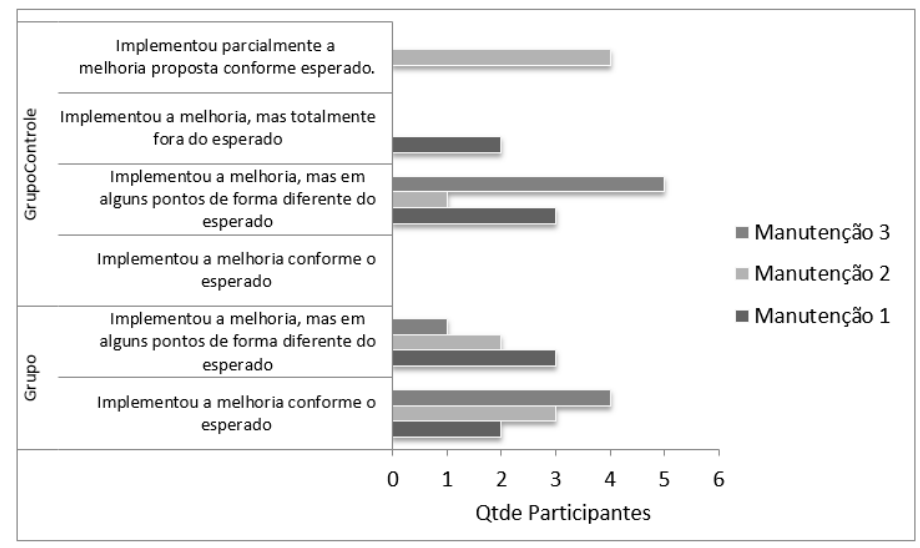

Figura 4. Qualidade Final da Implementação de Cada Manutenção

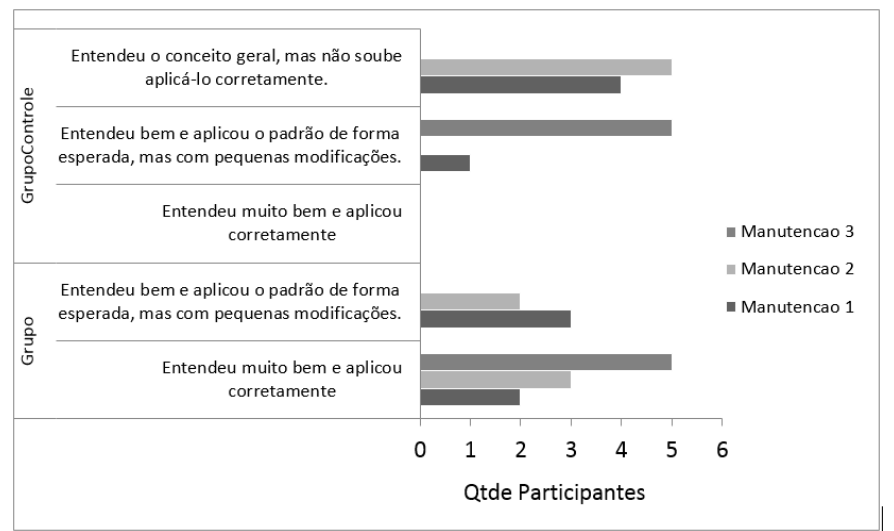

Figura 5. Nível de Entendimento em Cada uma das Manutenções Propostas

Considerando que quem teve acesso as unidades e objetos, teve uma solução com maior qualidade, um melhor entendimento e considerou as manutenções menos complexas, é um claro sinal de estes participantes conseguiram compreender melhor o padrão e tiveram uma maior aquisição do conhecimento.

\section{Discussão}

Este trabalho tem o objetivo de apresentar um ambiente colaborativo semântico, que possa auxiliar na aprendizagem organizacional em empresas desenvolvedoras de software. Além disso, foi apresentada uma questão de pesquisas, que deseja responder: "A organização das informações geradas nas empresas, por meio de ferramentas de edição colaborativa, em objetos e unidades de aprendizagem organizacionais, pode auxiliar na aprendizagem dos indivíduos em organizações desenvolvedoras de software?".

Afim de responder esta pergunta, uma versão inicial do ambiente foi implementada, e um experimento realizado. Considerando o experimento realizado, é evidenciado que o uso de ambiente pode contribuir em diversos aspectos aos membros de uma equipe de desenvolvimento de software. O ambiente age positivamente no auxílio ao compartilhamento do conhecimento e em uma melhor organização da 
informação. Estas características fazem com que os usuários possam ter um melhor entendimento e aprendizado, gerando benefícios como a resolução mais rápida e mais adequada das tarefas dependentes destes conhecimentos. Assim sendo, como descrito previamente, o uso do ambiente auxiliou na aquisição do conhecimento e os participantes conseguiram assimilar os conteúdos mais facilmente, adaptando-os para resolver os problemas propostos.

No entanto, a aprendizagem organizacional não depende exclusivamente da aprendizagem individual, pois é um conceito complexo, e pequenas divergências na sua conceitualização existem entre pesquisadores consolidados. Dessa maneira, a análise acerca deste tópico pode ser nebulosa e de difícil dedução. Indicativos claros devem ser devidamente organizados e apresentados, tomando como base fontes irrefutáveis, de maneira a conseguir reunir os elementos necessários para as argumentações e conclusões.

A fim de reunir estes elementos, uma discussão é apresentada a respeito de como o ambiente suporta os processos de conversão do conhecimento, propostos no processo de interação na criação do conhecimento de Nonaka e Konno (1998) do modelo de aprendizagem SECI. Este modelo é amplamente aceito e abrange todas as etapas necessárias para que a criação do conhecimento ocorra. Dessa maneira, é exposto como o ambiente proposto suporta cada um dos processos existentes nesta teoria:

-Socialização: O ambiente foi implementado com base em uma wiki, que pode auxiliar no processo de socialização. Além disso, a combinação de diversos materiais, organizados por um especialista pode auxiliar também neste processo.

-Externalização: O ambiente permite que os usuários codifiquem seu conhecimento e com a organização da SSEW de acordo com a MIC, este processo é facilitado para empresas desenvolvedoras de software.

-Internalização: Este processo pode ser facilitado pela nova organização do conteúdo em objetos e unidades de aprendizagem, auxiliando na aquisição do conhecimento.

- Combinação: Com a proposta de geração de unidades de aprendizagem de forma semi-automática, age diretamente na combinação, agregando conhecimento explícito em um conhecimento da organização. $\mathrm{O}$ conhecimento é contextualizado e agregado em uma unidade.

Portanto, considerando todo o exposto, há claros indícios que a organização das informações geradas nas empresas, por meio de ferramentas de edição colaborativa, em objetos e unidades de aprendizagem organizacionais, pode auxiliar na aprendizagem dos indivíduos em organizações desenvolvedoras de software.

\section{Considerações Finais}

A área de aprendizagem organizacional na engenharia de software é um tema explorado desde os anos 1990. No entanto, pela sua complexidade e amplitude, mesmo com o grande número de trabalhos desenvolvidos, diversas lacunas ainda permanecem. $\mathrm{Na}$ literatura são encontrados vários trabalhos que delineiam a aprendizagem em ambientes 
corporativos para situações específicas, e de alguma maneira pretendem apoiar a aprendizagem organizacional em empresas desenvolvedoras de software. Este trabalho tenta contribuir no assunto em questão, de forma a mostrar que um ambiente colaborativo semântico, baseado em objetos e unidades de aprendizagem organizacionais, podem auxiliar a fomentar a aprendizagem organizacional em empresas de desenvolvimento de software.

\section{Agradecimentos}

Agradecimento à Fundação Araucária (Fundação Araucária de Apoio ao Desenvolvimento Científico e Tecnológico do Estado do Paraná) pelo auxílio financeiro no desenvolvimento deste trabalho.

\section{Referências}

ADL (2004). “Advanced Distributed Learning. Scorm 4th Edition" http://www.adlnet.gov/Technologies/scorm/default.aspx.

Amorim, R. R.; Lama, M.; Sánchez, E.; Riera, A.; Vila, X. A.(2006) “A Learning Design Ontology based on the IMS Specification", Educational Technology \& Society, v. 9, p. 38-57.

Baader, F., Horrocks, I., Sattler, U. (2008) "Description Logics". Handbook of Knowledge Representation. Elsevier, c. 3, p. 135-180.

Berners-Lee, T.; Hendler, J.; Lassila, O. (2001) "The Semantic Web", Scientific American, v. 284, n. 5, p. 34-43.

Biasutti, M., El-Deghaidy, H. (2012) "Using Wiki in teacher education: Impact on knowledge management processes and student satisfaction", Computers \& Education, v.59, p861-872.

Capuano, N.; Miranda, S.; Orciuoli, F. (2009) "IWT: A Semantic Web-based Educational System". In: Workshop Of The Working Group On Ai \& E-Learning, p. 11-16.

FOAF (2010). "FOAF Vocabulary Specification 0.98”, http://xmlns.com/foaf/spec.

Gruber, T. (1995) "Toward principles for the design of ontologies used for knowledge sharing", International Journal of Human Computer Studies, v. 43, n.5-6, p. 907-928.

IEEE (2002) "IEEE Computer Society. Draft Standard for Learning Object Metadata", http://ltsc.ieee.org/wg12/files/LOM_1484_12_1_v1_Final_Draft.pdf.

IMS (2003) "IMS Global Learning Consortium Inc. Learning Design Specification", http://www.imsglobal.org/learningdesign/index.html.

IMS (2004) "IMS Global Learning Consortium Inc. IMS Content Packaging Information",http://www.imsglobal.org/content/packaging/cpv1p1p2/imscp_infov1p 1p2.html.

Menolli, A. L.; Malucelli, A.; Reinehr, S. (2011) "Towards a Semantic Social Collaborative Environment for Organizational Learning". In: International Conference on Information Technology And Applications (Icita), Sydney, p. 65-70. 
Menolli, A. L., Reinehr., S. , Malucelli, A. (2012a) "Ontology for Organizational Learning Objects based on LOM Standard". In: Proceedings of the Latin American Conference En Informática, Colombia.

Menolli, A. L., Reinehr, S., Malucelli, A. (2102b) Social Learning: Defining Learning Objects from Social Tools. In: Proceding of the International Conference WWW/Internet (ICWI), Madrid.

Menolli, A. L., Reinehr, S, Malucelli, A. (2013a) "Organizational Learning Applied to Software Engineering: A Systematic Review", International Journal of Software Engineering and Knowledge Engineering, v. 23, n. 8, p. 1153-1175.

Menolli, A. L., Reinehr, S, Malucelli, A. (2013b) "Improving Organizational Learning: Defining Units of Learning from Social Tools", Informatics in Education, v. 12 , n. 2, p.273.

Menolli, A. L., Pinto, S., Reinehr, S, Malucelli, A. (2014) "Unit of Organizational Learning Ontology based on LOM Standard and IMS Learning Design". In: International Conference on Formal Ontology in Information Systems, Brasil.

Nevis, E. C.; Di Bella, A.; Gould, J. M. (1995) "Understanding organizations as learning systems”, Sloan Management Review, v. 36, n.2, p. 73-85.

Nonaka, I., Konno, N. (1998) "The Concept of "Ba": Building a Foundation for Knowledge Creation", California Management Review, v. 40, n. 3, p. 40-54.

Ormrod, J.E. (1999) “Human learning”, Upper Saddle River: Prentice-Hall.

Rech J., Ras, E. (2008) "The Future of Learning Software Organizations: Semantics Collaboration - Aggregation". In: Workshop On Learning Software Organizations, Rome, Italy.

Rus, I.; Lindvall, M. (2002) “Knowledge management in software engineering”, IEEE Software v, 19, n. 3, p.26- 38.

Senge, P., Kleiner, A., Roberts, C., Ross, R., Smith, B. J. (1994) "The fifth discipline field book". New York: Doubleday.

Sicilia, M., Lytras, M. (2005) “The semantic learning organization", Learning Organization, v. 12, n. 5, p. 402-410.

Tseng, S.-M., Huang, J.-S. (2011) "The correlation between Wikipedia and knowledge sharing on job performance", Expert Systems with Applications, v. 38, n.5, p. 61186124.

Zouaq, A., Nkambou, R. (2009) "Enhancing Learning Objects with an Ontology-Based Memory", IEEE Transactions on Knowledge and Data Engineering, v. 21, n.6, p. 881-893. 